\title{
Quelques exemples d'applications du transfert d'électrons entre des conducteurs électroniques et des biomolécules
}

\author{
M. COMTAT
}

Laboratoire de Génie Chimique et Electrochimie, URA 192 du CNRS, Université Paul Sabatier, 118 Route de Narbonne, 31062 Toulouse, France

Les transferts électroniques sont impliqués dans de nombreux processus ou systèmes biologiques comme la photosynthèse, la respiration, les métabolismes, les réactions redox enzymatiques.

De ce fait, le transfert d'électrons entre des conducteurs électroniques et des biomolécules, réalisé directement ou grâce à l'emploi de médiateurs ou d'électrodes à surfaces modifiées, a de nombreuses applications dans des secteurs aussi diversifiés que la chimie fine, la biochimie analytique, la médecine, l'environnement, le contrôle et la régulation des procédés ou la conversion des énergies. Quelques exemples de la conséquence de la maîtrise de ces transferts hétérogènes sont présentés.

Le premier traite de la mise au point de biocapteurs électrochimiques à détection ampérométrique et des perspectives ouvertes par l'emploi de diverses méthodes d'activation du transfert électronique.

Le deuxième exemple concerne la mise au point de procédés bioélectrochimiques, notamment ceux de réduction de la méthémoglobine et de la régénération des coenzymes pyridiniques. L'application des principes de la biomimétique et le développement d'un génie chimique non conventionnel permettent de définir et de réaliser des appareillages pour la production de molécules à très haute valeur ajoutée.

Le troisième exemple porte sur la discussion des transferts d'électrons à l'intérieur de systèmes enzymatiques complexes comme les peroxydases, et des éventuelles applications dans le domaine du traitement bioélectrochimique de certains types d'effluents.

Electron transfers are encountered in various biological processes such as photosynthesis, respiration, metabolisms, enzymatic redox reactions. Electron transfers between metal or carbon and biomolecules, realized either directly either by means of various surface modifications have applications in fine chemistry, bioanalysis, medicine, environment, bioprocess regulations and energy conversion.

Some examples of applications of the mastery of these heterogeneous electron transfers are presented. The first deals with the proposal of biosensors with amperometric detection and describes some developments opened by the use of various methods of activation.

The second one is concerned with the development of bioelectrochemical processes specially for the methemoglobin reduction and the pyridinic coenzymes regeneration. Biomimetics and use of a non conventional chemical engineering enable the definition and the construction of new apparatus for the production of high value molecules.

The third example deals with the discussion of electron transfer inside peroxidases proposed to be the catalyst in various processes of bioelectrochemical treatments of wastes. 
La bioélectrochimie peut être définie, dans un premier temps, comme la discipline qui a en charge l'application des concepts, des théories et des méthodes de l'électrochimie, à l'étude des molécules et des systèmes biologiques organisés. Par extension, c'est aussi la partie de la physicochimie consacrée à la propagation des signaux électriques émis par les êtres vivants ou encore des réactions de ces derniers à l'application de champs électriques externes. Si l'on s'en tient à la première partie de cette définition, la bioélectrochimie représente un vaste domaine, puisque les molécules dont le rôle principal est d'assurer des relais électroniques ou des transports moléculaires d'espèces impliquées dans des réactions d'oxydo-réduction, sont très nombreuses [1-4]. En effet, les transferts d'électrons sont impliqués dans de nombreux processus ou systèmes biologiques comme la photosynthèse, la respiration, le métabolisme, les réactions redox enzymatiques, pharmacologiques ou radiobiologiques. Les molécules biologiques impliquées sont très diverses par leur masse, leur taille et la structure du centre redox qu'elles renferment. Les transferts électroniques hétérogènes entre des électrodes et ces différents types de biomolécules ont des vitesses très variées, qui dépendent de la nature du métal, de la taille, de la structure, de la fonction de la biomolécule et de sa disposition relative par rapport à la surface de l'électrode. Quand ils sont lents, il est possible d'avoir recours à des techniques de modification de surface pour assurer la promotion de ce transfert.

Le but de cet article est de présenter quelques méthodes couramment utilisées pour accélérer le transfert électronique électrode-biomolécule et de donner un aperçu des applications rendues possibles par la maîtrise et la modulation de ce transfert. Les exemples sont délibérément choisis dans des secteurs aussi variés que l'analyse en biologie clinique, la conception de nouveaux procédés biotechnologiques, la médecine ou la biologie moléculaire.

\section{Les biocapteurs électrochimiques à détection ampérométrique}

\subsection{Généralités}

Un biocapteur est un dispositif analytique dans lequel sont étroitement associés une couche de reconnaissance ionique ou moléculaire et un transducteur qui permet la conversion d'une concentration de substrat ou d'une activité biologique en un signal analogique. Dans le cas de l'électrochimie, le transducteur est une électrode et la détection peut être soit potentiométrique, soit ampérométrique. La précision est supérieure dans ce dernier cas, ce qui explique le grand nombre de biocapteurs ampérométriques actuellement mis au point.

La couche de reconnaissance moléculaire peut renfermer divers types de biocatalyseurs, enzymes, séquences d'enzymes, antigènes ou anticorps, microorganismes, fragments de cellule ou cellules entières. L'élément de reconnaissance peut être en solution au contact du transducteur, immobilisé dans un gel, greffé sur une membrane ou sur le transducteur luimême. Depuis la proposition, en 1962, de la première électrode à enzyme sélective du glucose, de nombreux biocapteurs électrochimiques à détection ampérométrique, aux multiples applications, ont été proposés [5-7].

L'exemple présenté concerne la réalisation d'un biocapteur à détection ampérométrique pour le dosage du L.lactate. 
La réaction mise en oeuvre est la suivante:

$$
\mathrm{CH}_{3} \mathrm{CHOHCOO}^{-}+2 \mathrm{Fe}(\mathrm{CN})_{6}{ }^{3-} \Leftrightarrow \mathrm{CH}_{3} \mathrm{COCOO}^{-}+2 \mathrm{Fe}(\mathrm{CN})_{6} 4^{-}+2 \mathrm{H}^{+}
$$

catalysée par une L.lactate déshydrogénase extraite d'Hansenula anomala. La détection se fait par suite de la réaction d'électrode:

$$
\mathrm{Fe}(\mathrm{CN})_{6} 4^{-} \Leftrightarrow \mathrm{Fe}(\mathrm{CN})_{6} 3^{-}+e
$$

réalisée sur une électrode portée à un potentiel constant (Fig.1).

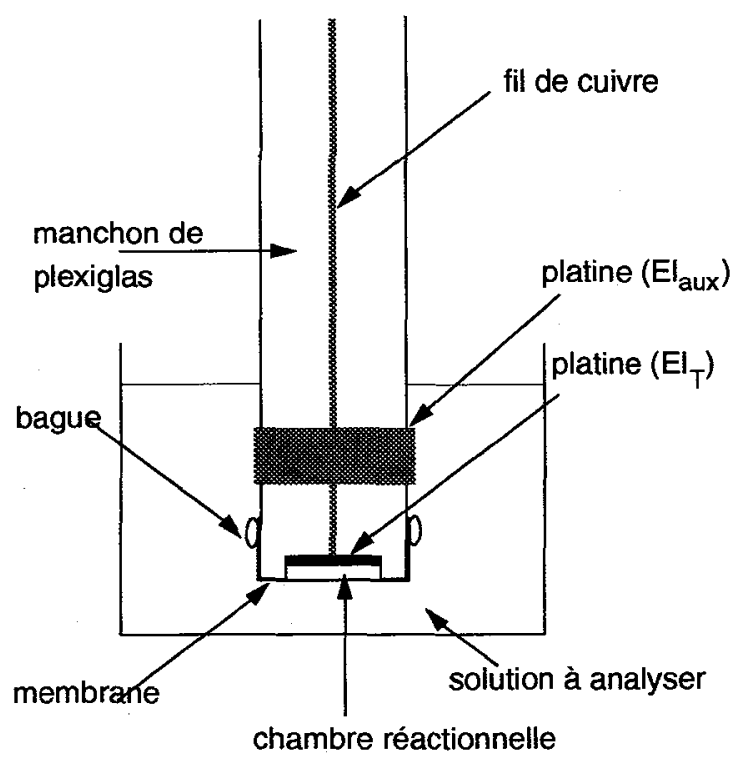

Figure 1 : schéma d'un capteur ampérométrique

Un tel capteur, qui utilise une enzyme en solution dans une chambre réactionnelle, permet, après optimisation des paramètres biochimiques, électrochimiques et géométriques, de doser le L.lactate avec une précision de $2 \%$ sur une gamme de concentration comprise entre 0,05 et $8 \mathrm{mM}$. Dans la mesure où l'échantillon analysé peut renfermer l'ion $\mathrm{Fe}(\mathrm{CN})_{6}{ }^{3-}$ en excès devant la concentration de $\mathrm{Fe}(\mathrm{CN})_{6}{ }^{4-}$ formé dans la chambre réactionnelle, il est possible d'employer un système dit à deux électrodes faiblement polarisées, entre lesquelles une différence de potentiel constante $(80 \mathrm{mV})$ est imposée grâce à une alimentation stabilisée. A l'heure actuelle, le débouché industriel de ce capteur concerne essentiellement la médecine du sport, mais il pourrait avoir bien d'autres applications dans l'analyse en biologie clinique, en chirurgie cardiovasculaire et dans les états d'urgence [8]. 


\subsection{Quelques tendances dans le développement d'un biocapteur}

Ce paragraphe est consacré à une analyse rapide des tendances de la recherche pour la mise au point de nouveaux biocapteurs électrochimiques, schématiquement divisées en quatre axes.

Le premier concerne l'emploi de microorganismes, de cellules ou de fragments de cellules, dont l'objectif principal est de contourner l'obstacle de la purification des enzymes. Bien entendu, ces capteurs, en général plus robustes que les capteurs à enzymes, sont moins sélectifs, répondent plus lentement et leurs créneaux d'applications sont surtout ceux de l'agroalimentaire et de l'environnement.

Le deuxième porte sur la mise en oeuvre des réactions antigène-anticorps, ce qui autorise la diversification des substrats détectés et la simplification du protocole du dosage immunologique.

Le troisième traite du développement de capteurs bi- ou multi-enzymatiques, dont les objectifs sont le dosage de nouveaux substrats, la diminution du seuil de détection si le produit d'une réaction enzymatique est alors substrat d'une deuxième enzyme, l'augmentation de la sélectivité si la deuxième enzyme est capable de détruire les substrats interférants ou de la sensibilité par l'exploitation du phénomène d'amplification enzymatique.

Le dernier exploite diverses méthodes d'activation du transfert électronique hétérogène. Pendant longtemps, il a été admis comme un dogme que ce transfert entre les conducteurs électroniques et les biomacromolécules était impossible. Les deux arguments avancés étaient d'une part la distance importante séparant l'électrode du centre redox souvent enfoui dans la structure protéique et la dénaturation de la biomolécule dans la double couche électrochimique. Une telle attitude a été préjudiciable au développement de la bioélectrochimie, ce qui est d'autant plus regrettable que ces arguments n'étaient pratiquement pas quantifiés. Par ailleurs, la notion même de biomolécule a été la source d'imprécisions et de confusions. Par exemple, les flavines font partie des systèmes oxydo-réducteurs les plus rapides étudiés à ce jour sur des électrodes de diverses natures.

Depuis une vingtaine d'années, l'amélioration des techniques de purification et l'emploi de méthodes électrochimiques plus sensibles ont permis de montrer que ce transfert électronique hétérogène était possible sans altération des propriétés fonctionnelles de la macromolécule. II faut reconnaître cependant qu'à part quelques exceptions, ce transfert est lent avec les biomacromolécules et il convient de l'accélérer pour avoir des capteurs aux temps de réponse convenables.

Dans la mise au point et le développement de biocapteurs électrochimiques, les méthodes d'activation sont utiles dans deux situations, l'augmentation de la sélectivité et la proposition d'électrodes dites sans cofacteur ou réactif. Dans le premier cas, il faut rechercher le potentiel à imposer le moins élevé dans le cas d'une oxydation ou le plus élevé dans le cas d'une réduction. Dans le deuxième cas, l'électrode est basée sur le transfert direct entre le conducteur électronique et la biomolécule.

* médiateurs en solution

L'une des possibilités pour accélérer le transfert électronique hétérogène est l'emploi de médiateurs redox en solution [9], moyen familier aux biologistes pour déterminer des potentiels normaux apparents à l'aide des techniques potentiométriques à courant nul. Ces 
médiateurs sont utilisés comme de véritables catalyseurs dans des mécanismes du type:

$$
\begin{aligned}
& \text { Mox + ne } \Leftrightarrow \text { Mred } \\
& \text { Mred + Ox } \Leftrightarrow \text { Red + Mox }
\end{aligned}
$$

dans lequel Mox / Mred représente le couple oxydo-réducteur du médiateur, Ox et Red les formes oxydée et réduite de la molécule biologique.

Dans ce mécanisme, la réaction électrochimique 1 est rapide sur une électrode donnée, la réaction 2 est spontanée et rapide. Il est préférable que la réaction 2 soit une réaction de transfert homogène pur, sans implication de phénomènes d'acido-basicité ou de complexation. D'autre part, la réaction 2 ne doit entraîner ni dénaturation ni modification de l'activité des molécules biologiques. Parmi les nombreux médiateurs proposés, il convient d'éviter, dans la mesure du possible, ceux qui donnent naissance à des intermédiaires radicalaires ou ceux qui réagissent avec l'oxygène, car ils sont susceptibles d'engendrer la formation de radicaux superoxyde $\mathrm{O}_{2}$ - ou hydroxyle $\mathrm{OH}$ - toxiques pour la plupart des molécules biologiques.

II existe de très nombreux travaux utilisant comme médiateurs des colorants et des quinones. Notons que la plupart des recherches de nature électrochimique réalisées ne se préoccupe pas du maintien de l'intégrité fonctionnelle de la molécule biologique après le transfert électronique. Il est pourtant important que les molécules biologiques conservent une bonne activité sur des temps parfois longs pour donner des capteurs utilisables industriellement.

II semble que les principes de la biomimétique ne soient pas encore suffisamment exploités dans le choix du médiateur. Une analyse détaillée des mécanismes de transfert d'électrons dans les systèmes organisés permet souvent de sélectionner le partenaire naturel de la molécule dans le transfert d'électrons et de reproduire ultérieurement sur l'électrode les mêmes conditions de transfert électronique. La compatibilité entre les diverses molécules est alors le garant du maintien de l'intégrité fonctionnelle de la macromolécule utilisée comme biocatalyseur.

${ }^{*}$ médiateurs fixés

Mais les médiateurs en solution contaminent l'échantillon à analyser, qui devient inutilisable pour d'autres déterminations. Pour pallier cet inconvénient, des fixations de médiateurs sont réalisées par différents moyens sur les électrodes. La méthode la plus simple est la fixation par adsorption forte. Dans le cas d'électrodes de carbone, l'oxydation anodique préalable, à des potentiels élevés, engendre sur la surface des composés quinoniques par suite d'oxydation électrochimique du carbone. Ces quinones peuvent servir de médiateurs, notamment pour l'oxydation des coenzymes pyridiniques.

Une autre méthode de fixation est l'inclusion du médiateur dans un polymère de surface. Bien entendu, les techniques d'électropolymérisation revêtent de nombreux avantages pour la confection de la couche biocatalytique, avec une immobilisation du catalyseur au contact du métal de l'électrode [10].

L'un des inconvénients essentiels de ces méthodes de fixation est la stabilité toute relative de la couche de biocatalyseur en surface de l'électrode. Cette instabilité se traduit par le rejet des groupes médiateurs dans la solution et l'on est confronté aux problèmes évoqués au paragraphe précédent. Une autre conséquence réside dans la mauvaise adhésion du film au conducteur électronique, et il s'en suit une augmentation importante de la résistance 
interfaciale et donc la nécessité d'une surtension de détection plus élevée.

L'une des solutions proposées est alors le greffage covalent sur la surface de l'électrode, par exemple par l'intermédiaire de divers groupements silanes.

Une place toute particulière doit être réservée aux composés du ferrocène, utilisés pour la première fois pour la catalyse de la réduction du cytochrome $c$. Depuis, cette molécule a donné naissance à toute une famille de capteurs pour le dosage du glucose et d'autres substrats.

II est possible de ranger dans cette catégorie les électrodes dans lesquelles des sels organiques conducteurs sont employés comme modificateurs de surface; en général ces composés, comme le tétrathiofulvalène, permettent le passage de l'électron entre l'électrode et la macromolécule [11].

* activateurs de surface

Une autre possibilité est l'utilisation d'activateurs de surface, molécules électroinactives dans le domaine de potentiel utilisé qui ne jouent en aucun cas le rôle de relais électronique dans le transfert conducteur électronique-biomolécule. Par contre, ces molécules sont bifonctionnelles, susceptibles de s'adsorber fortement sur la surface de l'électrode grâce à l'une des fonctions et capables d'être en interactions fortes avec la biomolécule grâce à l'autre fonction. La molécule la plus utilisée est la 4-4'-bipyridine, mais de nombreuses autres molécules ont depuis été proposées [12]

L'emploi d'ions divalents ou de complexes chargés positivement, non électroactifs, provoque l'accélération de certains transferts électroniques. L'exemple le plus typique est celui de l'électrode de dioxyde de ruthénium dont la charge de surface dépend du pH de la solution; suivant la charge de la biomolécule et la présence d'ions divalents, les constantes de transfert peuvent varier sur plusieurs décades [13-14] .

* transfert direct

La modulation du champ électrique local à l'in̂terface électrode - biomolécule permet d'obtenir également une promotion efficace du transfert électronique dans quelques cas particuliers. Les interactions électrostatiques entre le métal ou le carbone et la solution électrolytique conditionnent la position relative biomolécule - électrode et les distances entre l'électrode et les centres redox avant le transfert. De ce fait, elles influencent les constantes de vitesse du transfert électronique hétérogène et déterminent l'intensité du courant d'électrolyse. Les paramètres à prendre en compte sont le potentiel de l'électrode, la densité de charge superficielle, les charges globales ou locales de la biomolécule, mais aussi la composition ionique de la solution à proximité immédiate de la surface de l'électrode. Le nombre de ces paramètres fait qu'aucun traitement théorique n'est disponible et que l'approche est essentiellement empirique.

Des perturbations potentiodynamiques répétitives préalables permettent également, dans quelques cas particuliers, d'obtenir des transferts hétérogènes relativement rapides. Une première hypothèse simpliste est avancée: le choix d'un grand domaine de potentiel pour le balayage permet d'avoir des potentiels situés de part et d'autre du potentiel de charge nulle. Le fait que l'électrode prenne, à chaque balayage de potentiel, des valeurs positives et négatives de la charge de surface, optimise de façon empirique les interactions électrostatiques entre le conducteur électronique et la biomolécule. Cette dernière, au cours du 
temps, s'adsorbe très fortement à l'interface. Dans quelques cas particuliers, ces perturbations favorisent l'échange entre les molécules d'eau présentes dans la double couche et la biomolécule. La surface peut être rendue hydrophobe par le traitement préalable et la distance est réduite entre l'électrode et la biomolécule, ce qui favorise le transfert d'électrons. Ces mécanismes sont évoqués dans la réduction électrochimique directe de quelques enzymes [15].

La technique la plus récente est la modification d'enzyme, qui concerne surtout la glucose oxydase. La molécule peut être modifiée par des groupements flaviniques qui servent de relais électroniques entre l'électrode et la biomolécule [16-17]. D'autre part, différents polymères comme des polysiloxanes, fixés à l'électrode, permettent de réaliser des fils polymériques entre la molécule et l'électrode.

Enfin, le transfert électronique direct entre l'électrode et la biomolécule est possible, notamment si la molécule possède un équipement redox important. Il a été observé avec quelques molécules comme le cytochrome $a_{3}$, des ferredoxines, une lacticodéshydrogénase et une glucose oxydase, après un traitement électrochimique préalable [15] .

Ce type de transfert doit permettre, à terme, la mise au point et le développement de biocapteurs électrochimiques implantables ou directement utilisables dans le milieu biologique [18].

\section{Les procédés bioélectrochimiques}

Ces procédés mettent en oeuvre au moins une réaction de transfert électronique, direct ou indirect, entre une électrode et une biomolécule, en solution ou immobilisée en surface de l'électrode. Ils sont essentiellement orientés vers la préparation de produits chimiques ou biochimiques à haute valeur ajoutée. Les deux exemples présentés sont, à notre connaissance, les seuls à avoir atteint un stade de développement préindustriel.

\subsection{Procédé de réduction, de la méthémoglobine [19-20]}

La méthémoglobine (MetHb) est une hémoprotéine dans laquelle le fer est au degré d'oxydation +lll. Présente dans le sang à des teneurs de l'ordre de $1 \%$, elle ne joue aucun rôle dans le transport de l'oxygène qui est assuré par suite de l'équilibre entre la déoxyhémoglobine et l'oxyhémoglobine, où le fer est au degré d'oxydation +ll. Dans l'organisme, sa concentration est maintenue à une faible valeur grâce aux systèmes enzymatiques réducteurs présents dans le globule rouge.

Dans l'industrie, la méthémoglobine apparaît à la suite de divers traitements physiques du sang (lyophilisation, procédés de séparation par membranes ou par résines échangeuses d'ions). Sa réduction est, dans certains cas, une impérieuse nécessité, notamment pour la valorisation des effluents de l'industrie pharmaceutique, lors de la production de l'albumine ou des immunoglobulines. II est alors fondamental d'obtenir une déoxyhémoglobine ayant conservé ses propriétés fonctionnelles pour la fixation de l'oxygène. La courbe intensitépotentiel de réduction de la méthémoglobine, tracée avec une cellule en couche mince, ne montre pas de manifestation électrochimique autre que la réduction du solvant. Cependant, les spectres d'absorbance indique la réduction d'une partie des molécules présentes en solution. La lenteur de la réduction exclut la proposition d'un procédé et impose l'emploi d'un 
catalyseur. Le médiateur choisi est la flavine mononucléotide (FMN), dernier relais électronique dans le globule rouge, pour la réduction de la méthémoglobine. Cette molécule réagit très rapidement sur électrode de platine, avec des constantes de transfert électronique de l'ordre de $1 \mathrm{~cm} . \mathrm{s}^{-1}$. La réduction de la méthémoglobine devient alors rapide et le fait que la flavine soit son partenaire naturel assure la conservation de l'intégrité fonctionnelle de la molécule. A partir de ces résultats, en utilisant une démarche de génie chimique non conventionnelle, il a été possible de concevoir et de réaliser un appareil pour la mise en oeuvre du traitement de 100 litres de sang par jour. Cet appareil, représenté sur la figure 2, fonctionne à basse température pour éviter la prolifération bactérienne; il est conçu pour minimiser les contraintes de cisaillement qui conduiraient à la dénaturation des molécules biologiques. Enfin, il permet le travail en milieu strictement anaérobie grâce à des systèmes de bacs de stockage maintenus sous atmosphère inerte, en série avec les électrodes.

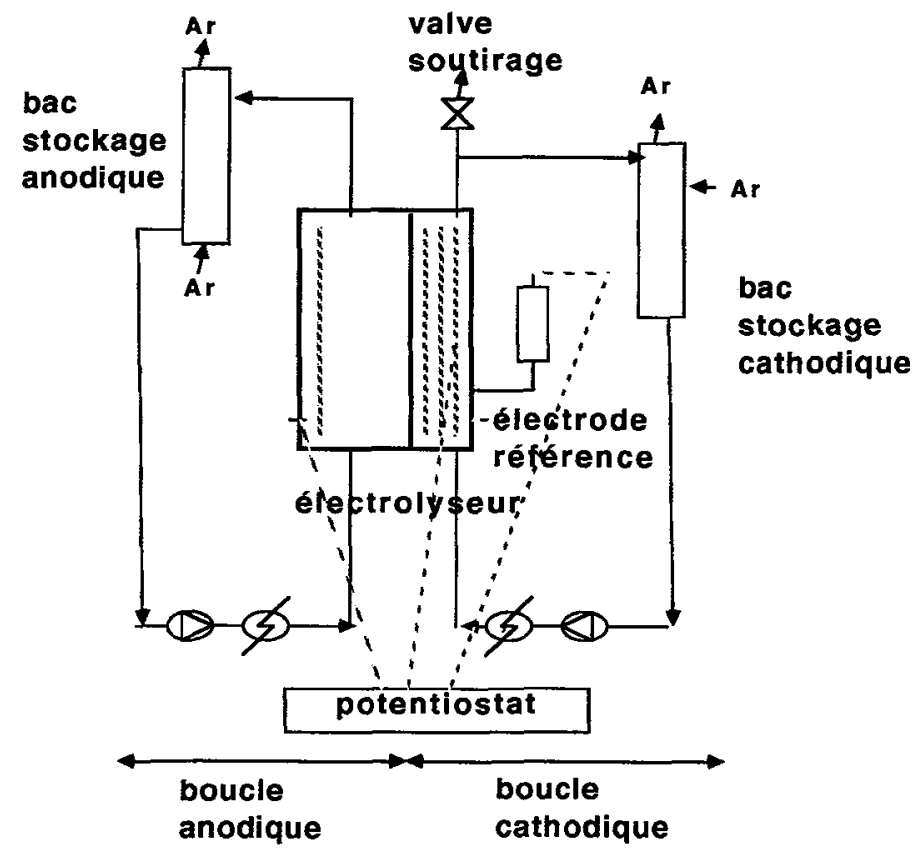

Figure 2 : Schéma de l'appareillage pour le procédé de réduction électrochimique de la méthémoglobine

1 pompe péristaltique

3 échangeur température $\left(4^{\circ} \mathrm{C}\right)$ ....... connection électrique

\subsection{Régénération des cofacteurs pyridiniques}

Le prix élevé des cofacteurs pyridiniques intervenant dans des réactions d'oxydoréduction catalysées par des enzymes exclut leur utilisation en quantité stoechiométrique et impose leur 
régénération. Malgré un nombre important de travaux mettant en oeuvre des méthodes très variées pour régénérer ces cofacteurs, aucun système ne s'est avéré satisfaisant, ce qui empêche le développement, dans le domaine de la chimie fine, de bioréacteurs intégrant des oxydoréductases. II faut reconnaître qu'à l'heure actuelle, les procédés enzymatiques sont les plus performants.

La régénération de la forme oxydée $\mathrm{NAD}^{+}$par voie électrochimique est réalisable sur des électrodes de type feutre de carbone incorporées dans des réacteurs de laboratoire traitant quelques dizaines de centimètres cubes de solution [21].

Dans le cas de la régénération électrochimique de la forme réduite NADH, l'obstacle majeur est l'apparition d'un intermédiaire radicalaire susceptible d'une dimérisation rapide. Une voie originale semble s'ouvrir avec la possibilité de catalyser la réaction de réduction par une hydrogénase [22], sans médiateur en solution. Le rendement est supérieur à $99 \%$ et la vitesse est suffisamment grande pour que le procédé industriel soit envisagé. Des fréquences de régénération de NADH de l'ordre de 450 par heure sont d'ores et déjà obtenues avec un réacteur de laboratoire [23]. L'une des conséquences importantes dans la mise au point du procédé électrochimique de régénération de NADH est l'effet de l'application du potentiel sur l'activité de l'enzyme. La figure 3 montre qu'il est possible d'obtenir, après application d'un champ électrique, une solution enzymatique plus active que la solution mère et présentant une meilleure résistance à la désactivation thermique. L'une des explications possibles est que le potentiel réducteur imposé à la molécule la protège contre les effets des intermédiaires radicalaires de l'oxygène auxquels elle est particulièrement sensible.

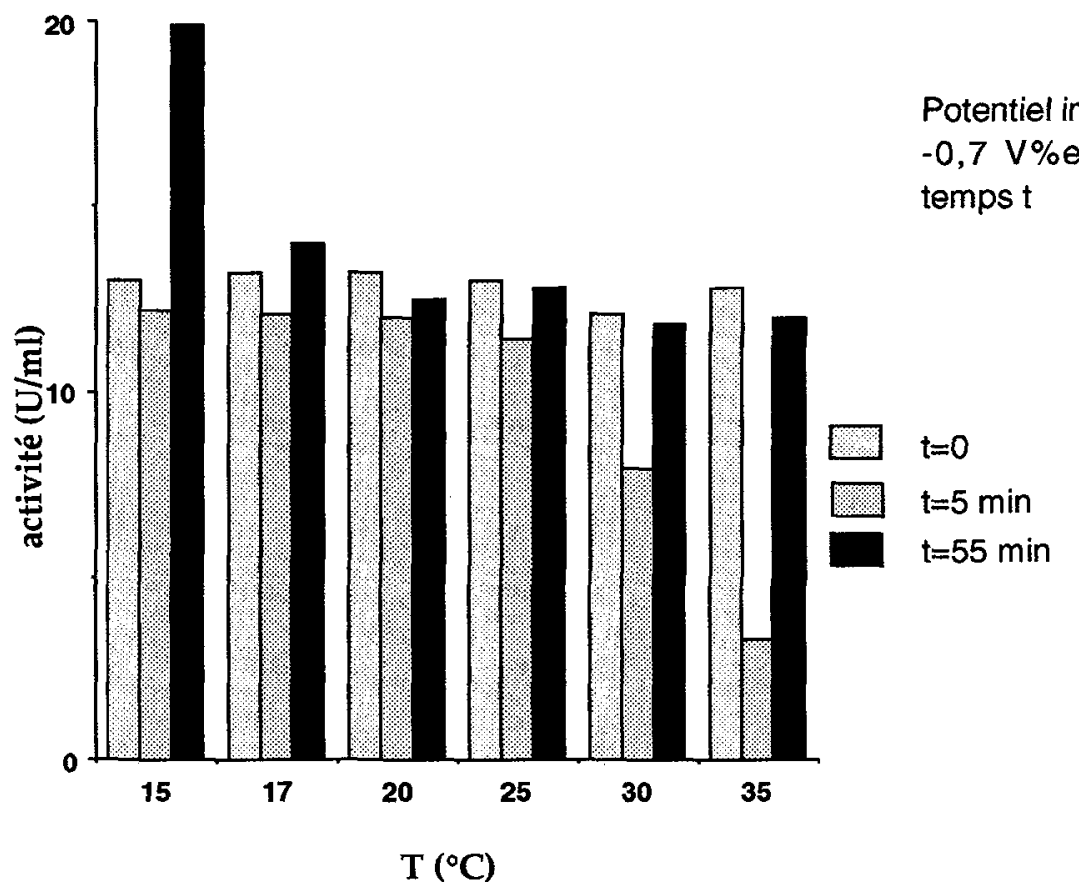

Figure 3: Evolution de l'activité de l'hydrogénase d'Alcaligenes eutrophus en fonction de la température et de l'application d'un champ électrique 


\section{L'électrochimie des peroxydases}

Les peroxydases sont des enzymes qui catalysent des réactions d'oxydation par le peroxyde d'hydrogène du type: $\quad 2 \mathrm{AH}+\mathrm{H}_{2} \mathrm{O}_{2} \rightarrow \mathrm{A}_{2}+2 \mathrm{H}_{2} \mathrm{O}$

Ces molécules hémiques, à l'état natif, ont un fer au degré d'oxydation III. L'action du peroxyde d'hydrogène sur l'enzyme conduit au composé I dans lequel le fer est au degré d'oxydation formel IV, qui possède un radical cation. Une première molécule de substrat réduit le composé I en composé II par neutralisation du radical cation; la deuxième molécule de substrat réduit de façon monoélectronique le composé II en ferriperoxydase native. Un autre composé intermédiaire, le composé III, peut apparaître, soit par suite de la fixation de l'oxygène sur la ferroperoxydase (fer au degré II), soit par réaction de l'anion superoxyde sur la ferriperoxydase.
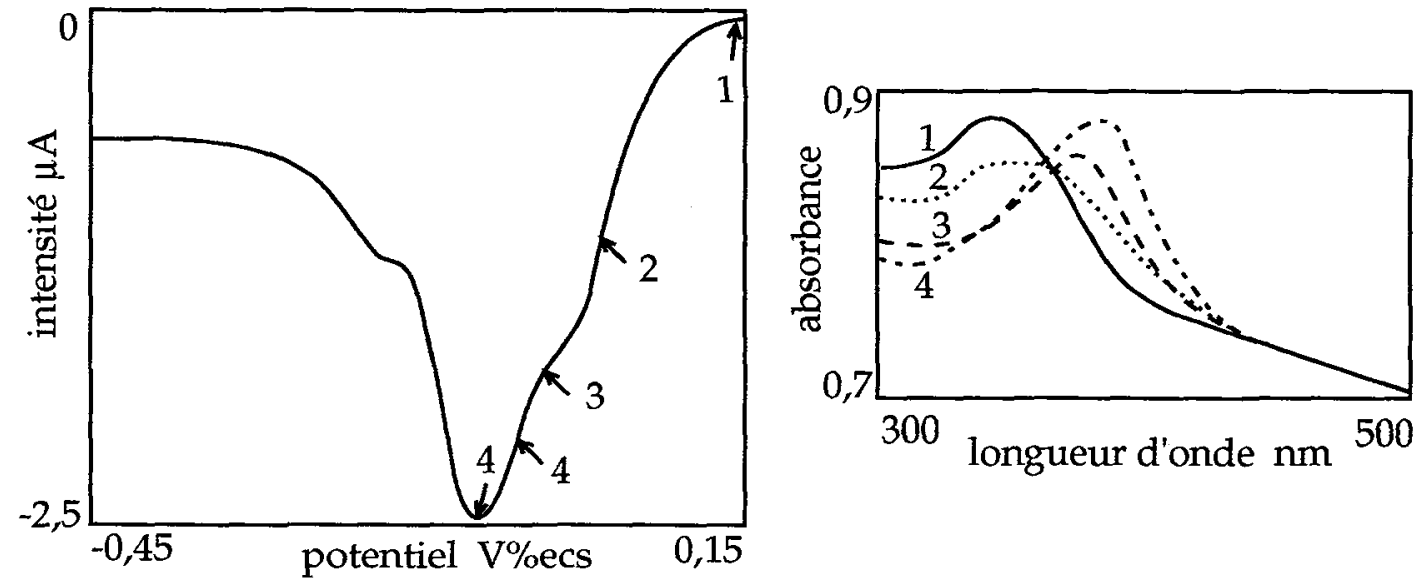

Figure 4 : Spectroélectrochimie en couche mince de la peroxydase de raifort $\mathrm{pH}=8,2$; concentration: $70 \mu \mathrm{M}$; vitesse de balayage des potentiels: $15 \mathrm{mV} \mathrm{min}^{-1}$

Bien que la diversité des degrés d'oxydation du fer dans cette molécule justifie une étude électrochimique, peu de travaux sont rencontrés dans la bibliographie. Pourtant, il est possible de préparer par voie électrochimique les divers composés intermédiaires évoqués précédemment. La figure 4 montre par exemple le résultat d'une étude par spectroélectrochimie en couche mince réalisée sur cathode de platine, avec un mélange de ferriperoxydase et d'oxygène. Les spectres permettent d'identifier, suivant les potentiels appliqués, l'apparition du composé III, du composé II et de la ferriperoxydase. Pour des potentiels plus cathodiques, la réduction en ferroperoxydase nécessite la présence de flavine mononucléotide qui joue le rôle de médiateur [24]. Un tel exemple montre qu'il est possible de moduler, par voie électrochimique, l'activité enzymatique des peroxydases.

D'autre part, l'utilisation simultanée d'une peroxydase et d'une électrode, en milieu oxygéné, permet d'engendrer le composé I et de l'impliquer dans des réactions d'oxydation du phénol conduisant à des polymères. Il y a là, sans doute, une voie à explorer, pour la mise au point de réacteurs électrochimiques, afin de traiter des effluents pollués par des phénols. 


\section{Conclusion}

Les quelques exemples ci-dessus, pris parmi les résultats du laboratoire de génie chimique et électrochimie, montrent que la mise au point et le développement des applications de la bioélectrochimie procèdent souvent de la même démarche. La première étape réside dans le choix d'un moyen pour accélérer le transfert électronique électrode-biomolécule. L'éventail des possibilités est maintenant relativement étendu. Cependant, dans la mesure où l'efficacité doit être maintenue sur de longues périodes, il faut conserver l'intégrité fonctionnelle de la molécule; cette contrainte est souvent surmontée en faisant appel à la biomimétique. En effet, l'analyse du fonctionnement de la biomolécule dans le système biologique organisé permet souvent de choisir les conditions d'interface ou les médiateurs les mieux adaptés pour obtenir un transfert rapide, sans dénaturation, sur des durées importantes. La deuxième étape traite de la mise en oeuvre optimale de la biomolécule dans le réacteur d'utilisation. Une réflexion sur les problèmes de couplage entre les phénomènes de transport, les réactions chimiques et électrochimiques, est nécessaire pour définir le meilleur mode de confinement ou d'immobilisation du biocatalyseur près de l'électrode. Cette étape est souvent génératrice d'innovation au niveau de l'appareillage. La troisième étape est celle de la modélisation et du dimensionnement des réacteurs et des appareillages associés.

II est également possible d'envisager l'utilisation de l'électrochimie pour concevoir de nouveaux produits destinés à lutter contre le vieillissement cellulaire $[25,26]$.

La méthodologie décrite peut être appliquée, non plus à des systèmes moléculaires, mais à des cellules ou des microorganismes, ce qui ouvre la voie à de nouveaux procédés bioélectrochimiques, pour le contrôle et la régulation de fermenteurs ou d'appareils de cultures cellulaires, également pour la conversion de l'énergie biochimique en énergie électrique [27].

\section{Références}

1 - S.SRINIVASAN, Y.A.CHIZMADZHEV, J.O.M.BOCKRIS, B.E.CONWAY et E.YEAGER, Comprehensive treatise of electrochemistry, Vol.10, Plenum press New York, 1985.

2 - F.GUTMANN et H.KEYSER, Modern bioelectrochemistry, Plenum Press New York, 1987.

3 - L.I.BOGUSLAVSKY dans Modern aspects of electrochemistry, $n^{\circ} 18$, Plenum Press New York, 1986, 113-168, R.E.WHITE, J.O.M.BOCKRIS et B.E.CONWAY Ed.

4 - R.PLONSEY et R.C.BARR, Bioelectricity: a quantitative approach, Plenum Press, New York, 1988.

5 - A.P.F. TURNER, I. KARUBE AND G.S. WILSON, Biosensors: Fundamentals and Applications, Oxford University Press, Oxford, 1987.

6 - F. SCHELLER AND F. SCHUBERT, Biosensors, Elsevier, New-York, 1990.

7 - L.BLUM et P.R.COULET, Biosensors: Principles and Applications, Marcel Dekker, New York, 1991.

8 - M.COMTAT, L'actualité Chimique, 1992, 1,115-123.

9 - M.L.FULTZ et R.A.DURST, Anal.Chim.Acta, 1982, 140,1-18.

10 - R.G.LINFORD, Electrochemical Science and Technology of polymers, Elsevier, New York, 1987.

11 - J.KULYS et R.D.SCHMID, Biosensors and Bioelectronics, 1991,6,43-48. 
12 - H.A.O.HILL et G.A.LAWRANCE, J.Electroanal.Chem., 1989,270,309-318.

13 - F.A.ARMSTRONG, H.A.O.HILL et N.J.WALTON, Quart.Rev.Biophys., 1986, 18,261-267.

14 - F.A.ARMSTRONG, H.A.O.HILL et N.J.WALTON, Acc.Chem.Res., 1988,21,407-412.

15 - H.DURLIAT et M.COMTAT, Anal.Chem., 1982,54,856-861.

16 - Y.DEGANI et A.HELLER, J.Am.Chem.Soc., 1988, 110,2615-2620.

17 - A.HELLER, Acc.Chem.Res., 1990,23,128-134.

18 - J.JANATA, Anal.Chem., 1992,64,196R-219R.

19 - H.DURLIAT et M.COMTAT, J.Biol.Chem., 1987,262,11497-11500.

20 - P.LABRUNE et A.BERGEL, Chem. Eng.Sci., 1992,47,1219-1238.

21 - J.M.LAVAL, J.MOIROUX et C.BOURDILLON, Biotechnol.Bioeng., 1991,38,788-792.

22 - H.DURLIAT, M.COMTAT et J.L.SERIS, Anal.Lett., 1991,24,1471-1482.

23 - J.CANTET, A.BERGEL et M.COMTAT, Bioelectrochem.Bioenerg., 1992,27,475-486.

24 - H.DURLIAT, A.COURTEIX et M.COMTAT, Bioelectrochem.Bioenerg., 1989,22,197-209.

25 - I.EMERIT et B.CHANCE, Free radicals and aging, Birkhauser Verlag, Bâle 1992.

26 - H.SIES, Oxidative stress, Academic Press, New York, 1985.

27 - H.P.BENNETTO dans Life Chemistry Reports, A.M.MICHELSON et J.V.BANNISTER Ed, Harwood London, 1984, 2,363-451. 